\title{
ASYMPTOTIC APPROXIMATIONS OF EIGEN- FUNCTIONS FOR REGULAR STURM-LIOUVILLE PROBLEMS WITH EIGENVALUE PARAMETER IN THE BOUNDARY CONDITION FOR INTEGRABLE POTENTIAL
}

\author{
HASKIZ COŞKUN and AYŞE KABATAŞ
}

\begin{abstract}
In this paper we obtain asymptotic estimates of eigenfunctions for regular Sturm-Liouville problems having the eigenparameter in the boundary condition without smoothness conditions on the potential.
\end{abstract}

\section{Introduction}

In this paper we consider the boundary value problem

$$
\begin{gathered}
\tau y:=-y^{\prime \prime}+q y=\lambda y, q(t) \quad \text { is integrable on } \quad[a, b], \\
a_{1} y(a)-a_{2} y^{\prime}(a)=\lambda\left(a_{3} y(a)-a_{4} y^{\prime}(a)\right), \quad a_{1}, a_{2}, a_{3}, a_{4} \in \mathrm{R}, \\
y(b) \cos \beta+y^{\prime}(b) \sin \beta=0, \quad \beta \in[0, \pi)
\end{gathered}
$$

where $\lambda$ is a real parameter. It is shown by Walter [16] that this problem is a self-adjoint problem if the relation

$$
\delta:=\left|\begin{array}{ll}
a_{3} & a_{1} \\
a_{4} & a_{2}
\end{array}\right|>0
$$

holds. Problems of this type arise upon separation of variables in the one dimensional wave and heat equations. The cases $a_{4}=0(\beta=0, \beta \neq 0)$ and $a_{4} \neq 0(\beta=0, \beta \neq 0)$ are considered in [5] where $q(t)$ is continuous in $[a, b]$. Fulton's approach in [5] is based on making use of solutions of (1) and employing the residue calculus to give more direct proof of the convergence properties of the eigenfunction expansion of (1)-(3). Some other related results can be found in [1], [6], [8], [9], [10], [11], [12], [13], [14], [15], [16]. 
The aim of this paper is to obtained asymptotic approximations for the eigenfunctions of (1)-(3) including the above mentioned cases $\left(a_{4}=0, a_{4} \neq\right.$ 0 ) where $q(t)$ is integrable on $[a, b]$. We use similar approach in [4], [7] to derive these approximations by making use of solutions of (1) defined with the initial conditions and asymptotic approximations of the eigenvalues of (1)-(3) obtained in [2].

AcKnowledgements. The first author is grateful to Professor Bernard J. Harris for introducing her to the field. Both authors record their gratitude to the referee for his (or her) careful reading of the paper.

\section{The Results}

We define two solutions, $\Psi(x, \lambda)$ and $\Phi(x, \lambda)$ of (1)-(3) with the initial conditions

$$
\psi(a, \lambda)=a_{4} \lambda-a_{2}, \Psi^{\prime}(a, \lambda)=a_{3} \lambda-a_{1},
$$

and

$$
\Phi(b, \lambda)=\sin \beta, \Phi^{\prime}(b, \lambda)=-\cos \beta .
$$

THEOREM 2.1. Let $\Psi(x, \lambda)$ be the solution of (1) satisfying (4). Then

(i) $a_{4} \neq 0$

(6) $\Psi(x, \lambda)=\frac{a_{4} \lambda-a_{2}}{\cos \left\{\tan ^{-1}\left[F_{1}(a, \lambda)\right]\right\}} \exp \left(\int_{a}^{x} S(t, \lambda) d t\right)$

$$
\times \cos \left\{\tan ^{-1}\left[F_{1}(a, \lambda)\right]+\int_{a}^{x} T(t, \lambda) d t\right\},
$$

where

$$
F_{1}(a, \lambda):=\frac{1}{T(a, \lambda)}\left[S(a, \lambda)-\frac{a_{3} \lambda-a_{1}}{a_{4} \lambda-a_{2}}\right] .
$$

(ii) $a_{4}=0$

(8) $\Psi(x, \lambda)=-\frac{a_{2}}{\cos \left\{\cot ^{-1}\left[F_{2}(a, \lambda)\right]\right\}} \exp \left(\int_{a}^{x} S(t, \lambda) d t\right)$

$$
\times \cos \left\{\cot ^{-1}\left[F_{2}(a, \lambda)\right]+\int_{a}^{x} T(t, \lambda) d t\right\},
$$


where

$$
F_{2}(a, \lambda):=\frac{a_{2} T(a, \lambda)}{a_{3} \lambda-a_{1}+a_{2} S(a, \lambda)} .
$$

Theorem 2.2. Let $\Phi(x, \lambda)$ be the solution of (1) satisfying (5). Then

(i) $\beta \neq 0$

(10)

$$
\begin{aligned}
& \Phi(x, \lambda)=\frac{\sin \beta}{\exp \left(\int_{a}^{b} S(t, \lambda) d t\right) \cos \left\{\tan ^{-1}\left[F_{3}(b, \lambda)\right]\right\}} \exp \left(\int_{a}^{x} S(t, \lambda) d t\right) \\
& \times \cos \left\{\tan ^{-1}\left[F_{3}(b, \lambda)\right]-\int_{x}^{b} T(t, \lambda) d t\right\},
\end{aligned}
$$

where

$$
F_{3}(b, \lambda):=\frac{S(b, \lambda)+\cot \beta}{T(b, \lambda)}
$$

(ii) $\beta=0$

(12) $\Phi(x, \lambda)=\frac{1}{\exp \left(\int_{a}^{b} S(t, \lambda) d t\right) T(b, \lambda)} \exp \left(\int_{a}^{x} S(t, \lambda) d t\right)$

$$
\times \cos \left\{\frac{\pi}{2}-\int_{x}^{b} T(t, \lambda) d t\right\} .
$$

THEOREM 2.3. Solution of (1) corresponding to (4) satisfies, as $\lambda \longrightarrow \infty$

(i) $a_{4} \neq 0$

(13) $\Psi(x, \lambda)=a_{4} \lambda \cos \left[\lambda^{\frac{1}{2}}(x-a)\right]+\lambda^{\frac{1}{2}}\left[a_{3}+\frac{a_{4}}{2} \int_{a}^{x} q(t) d t\right]$

$$
\times \sin \left[\lambda^{\frac{1}{2}}(x-a)\right]+O\left(\lambda^{\frac{1}{2}} \eta(\lambda)\right),
$$

(ii) $a_{4}=0$

(14) $\Psi(x, \lambda)=a_{3} \lambda^{\frac{1}{2}} \sin \left[\lambda^{\frac{1}{2}}(x-a)\right]-\left[a_{2}+\frac{a_{3}}{2} \int_{a}^{x} q(t) d t\right]$

$$
\times \cos \left[\lambda^{\frac{1}{2}}(x-a)\right]+O(\eta(\lambda))
$$


where $\eta(\lambda):=\sup _{a \leq t \leq b} F(t, \lambda)$ with

$$
F(t, \lambda):= \begin{cases}\frac{\left|\int_{t}^{b} e^{2 i \lambda^{\frac{1}{2}} x} q(x) d x\right|}{\int_{t}^{b}|q(x)| d x} & \text { if } \int_{t}^{b}|q(x)| d x \neq 0, \\ 0 & \text { if } \int_{t}^{b}|q(x)| d x=0 .\end{cases}
$$

We note that $\eta(\lambda) \longrightarrow 0$ as $\lambda \rightarrow \infty[2]$.

Theorem 2.4. Solution of (1) corresponding to (5) satisfies, as $\lambda \longrightarrow \infty$

(i) $\beta \neq 0$

(15) $\Phi(x, \lambda)=\sin \beta \cos \left[\lambda^{\frac{1}{2}}(b-x)\right]+\lambda^{-\frac{1}{2}}\left[\cos \beta+\frac{\sin \beta}{2} \int_{x}^{b} q(t) d t\right]$

$$
\times \sin \left[\lambda^{\frac{1}{2}}(b-x)\right]+O\left(\lambda^{-\frac{1}{2}} \eta(\lambda)\right),
$$

(ii) $\beta=0$

$$
\begin{aligned}
\Phi(x, \lambda)=\lambda^{-\frac{1}{2}} \sin \left[\lambda^{\frac{1}{2}}(b-x)\right]- & \frac{1}{2} \lambda^{-1}\left[\int_{x}^{b} q(t) d t\right] \\
& \times \cos \left[\lambda^{\frac{1}{2}}(b-x)\right]+O\left(\lambda^{-1} \eta(\lambda)\right) .
\end{aligned}
$$

Our results include the following four distinct cases concerning $a_{4}$ and $\beta$ as pointed out in [5]. These are $a_{4} \neq 0, \beta \neq 0 ; a_{4} \neq 0, \beta=0 ; a_{4}=0, \beta \neq 0$ and $a_{4}=0, \beta=0$.

THEOREM 2.5. The asymptotic formula for the eigenfunctions of (1) and (4) satisfies, as $n \longrightarrow \infty$

(i) $a_{4} \neq 0, \beta \neq 0$

$$
\begin{aligned}
\Psi\left(x, \lambda_{n}\right) & =a_{4} \frac{(n+1)^{2} \pi^{2}}{(b-a)^{2}} \cos \left[\frac{(n+1) \pi(x-a)}{b-a}\right]+\frac{(n+1) \pi}{b-a} \\
& \times\left[a_{3}+\frac{a_{4}}{2} \int_{a}^{x} q(t) d t\right] \sin \left[\frac{(n+1) \pi(x-a)}{b-a}\right]+O(n \eta(n)),
\end{aligned}
$$

(ii) $a_{4} \neq 0, \beta=0$

(18) $\Psi\left(x, \lambda_{n}\right)=a_{4} \frac{(2 n+3)^{2} \pi^{2}}{4(b-a)^{2}} \cos \left[\frac{(2 n+3) \pi(x-a)}{2(b-a)}\right]+\frac{(2 n+3) \pi}{2(b-a)}$

$$
\times\left[a_{3}+\frac{a_{4}}{2} \int_{a}^{x} q(t) d t\right] \sin \left[\frac{(2 n+3) \pi(x-a)}{2(b-a)}\right]+O(n \eta(n)),
$$


(iii) $a_{4}=0, \beta \neq 0$

(19) $\Psi\left(x, \lambda_{n}\right)=a_{3} \frac{(2 n+3) \pi}{2(b-a)} \sin \left[\frac{(2 n+3) \pi(x-a)}{2(b-a)}\right]$

$$
-\left[a_{2}+\frac{a_{3}}{2} \int_{a}^{x} q(t) d t\right] \cos \left[\frac{(2 n+3) \pi(x-a)}{2(b-a)}\right]+O(\eta(n)),
$$

(iv) $a_{4}=0, \beta=0$

(20) $\Psi\left(x, \lambda_{n}\right)=a_{3} \frac{(n+2) \pi}{b-a} \sin \left[\frac{(n+2) \pi(x-a)}{b-a}\right]$

$$
-\left[a_{2}+\frac{a_{3}}{2} \int_{a}^{x} q(t) d t\right] \cos \left[\frac{(n+2) \pi(x-a)}{b-a}\right]+O(\eta(n)) .
$$

THEOREM 2.6. The asymptotic formula for the eigenfunctions of (1) and (5) satisfies, as $n \longrightarrow \infty$

(i) $a_{4} \neq 0, \beta \neq 0$

(21) $\Phi\left(x, \lambda_{n}\right)=\sin \beta \cos \left[\frac{(n+1) \pi(b-x)}{b-a}\right]+\frac{b-a}{(n+1) \pi}$

$$
\times\left[\cos \beta+\frac{\sin \beta}{2} \int_{x}^{b} q(t) d t\right] \sin \left[\frac{(n+1) \pi(b-x)}{b-a}\right]+O\left(n^{-1} \eta(n)\right),
$$

(ii) $a_{4} \neq 0, \beta=0$

(22) $\Phi\left(x, \lambda_{n}\right)=\frac{2(b-a)}{(2 n+3) \pi} \sin \left[\frac{(2 n+3) \pi(b-x)}{2(b-a)}\right]-\frac{2(b-a)^{2}}{(2 n+3)^{2} \pi^{2}}$

$$
\times\left[\int_{x}^{b} q(t) d t\right] \cos \left[\frac{(2 n+3) \pi(b-x)}{2(b-a)}\right]+O\left(n^{-2} \eta(n)\right),
$$

(iii) $a_{4}=0, \beta \neq 0$

(23) $\Phi\left(x, \lambda_{n}\right)=\sin \beta \cos \left[\frac{(2 n+3) \pi(b-x)}{2(b-a)}\right]+\frac{2(b-a)}{(2 n+3) \pi}$

$$
\times\left[\cos \beta+\frac{\sin \beta}{2} \int_{x}^{b} q(t) d t\right] \sin \left[\frac{(2 n+3) \pi(b-x)}{2(b-a)}\right]+O\left(n^{-1} \eta(n)\right),
$$


(iv) $a_{4}=0, \beta=0$

(24) $\Phi\left(x, \lambda_{n}\right)=\frac{b-a}{(n+2) \pi} \sin \left[\frac{(n+2) \pi(b-x)}{b-a}\right]-\frac{(b-a)^{2}}{2(n+2)^{2} \pi^{2}}$

$$
\times\left[\int_{x}^{b} q(t) d t\right] \cos \left[\frac{(n+2) \pi(b-x)}{b-a}\right]+O\left(n^{-2} \eta(n)\right) .
$$

For $a=0, b=\pi$ we get the following corollaries:

COROLlary 2.7. The eigenfunctions $\Psi\left(x, \lambda_{n}\right)$ of (1) corresponding to (4) satisfy, as $n \longrightarrow \infty$

(i) $a_{4} \neq 0, \beta \neq 0$

(25) $\Psi\left(x, \lambda_{n}\right)=a_{4}(n+1)^{2} \cos [(n+1) x]+(n+1)$

$$
\times\left[a_{3}+\frac{a_{4}}{2} \int_{0}^{x} q(t) d t\right] \sin [(n+1) x]+O(n \eta(n)),
$$

(ii) $a_{4} \neq 0, \beta=0$

(26) $\Psi\left(x, \lambda_{n}\right)=a_{4} \frac{(2 n+3)^{2}}{4} \cos \left[\frac{(2 n+3) x}{2}\right]+\frac{(2 n+3)}{2}$

$$
\times\left[a_{3}+\frac{a_{4}}{2} \int_{0}^{x} q(t) d t\right] \sin \left[\frac{(2 n+3) x}{2}\right]+O(n \eta(n)),
$$

(iii) $a_{4}=0, \beta \neq 0$

(27) $\Psi\left(x, \lambda_{n}\right)=a_{3} \frac{(2 n+3)}{2} \sin \left[\frac{(2 n+3) x}{2}\right]$

$$
-\left[a_{2}+\frac{a_{3}}{2} \int_{0}^{x} q(t) d t\right] \cos \left[\frac{(2 n+3) x}{2}\right]+O(\eta(n)),
$$

(iv) $a_{4}=0, \beta=0$

(28) $\Psi\left(x, \lambda_{n}\right)=a_{3}(n+2) \sin [(n+2) x]$

$$
-\left[a_{2}+\frac{a_{3}}{2} \int_{0}^{x} q(t) d t\right] \cos [(n+2) x]+O(\eta(n)) .
$$

Corollary 2.8. The eigenfunctions $\Phi\left(x, \lambda_{n}\right)$ of (1) corresponding to (5) satisfy, as $n \longrightarrow \infty$ 
(i) $a_{4} \neq 0, \beta \neq 0$

(29) $\Phi\left(x, \lambda_{n}\right)=\sin \beta \cos [(n+1)(\pi-x)]+\frac{1}{(n+1)}$

$$
\times\left[\cos \beta+\frac{\sin \beta}{2} \int_{x}^{\pi} q(t) d t\right] \sin [(n+1)(\pi-x)]+O\left(n^{-1} \eta(n)\right),
$$

(ii) $a_{4} \neq 0, \beta=0$

(30) $\Phi\left(x, \lambda_{n}\right)=\frac{2}{(2 n+3)} \sin \left[\frac{(2 n+3)(\pi-x)}{2}\right]-\frac{2}{(2 n+3)^{2}}$

$$
\times\left[\int_{x}^{\pi} q(t) d t\right] \cos \left[\frac{(2 n+3)(\pi-x)}{2}\right]+O\left(n^{-2} \eta(n)\right),
$$

(iii) $a_{4}=0, \beta \neq 0$

(31)

$$
\begin{aligned}
& \Phi\left(x, \lambda_{n}\right)=\sin \beta \cos \left[\frac{(2 n+3)(\pi-x)}{2}\right]+\frac{2}{(2 n+3)} \\
& \times\left[\cos \beta+\frac{\sin \beta}{2} \int_{x}^{\pi} q(t) d t\right] \sin \left[\frac{(2 n+3)(\pi-x)}{2}\right]+O\left(n^{-1} \eta(n)\right),
\end{aligned}
$$

(iv) $a_{4}=0, \beta=0$

$$
\begin{aligned}
\Phi\left(x, \lambda_{n}\right)=\frac{1}{(n+2)} \sin [(n+2)(\pi-x)]-\frac{1}{2(n+2)^{2}} \\
\times\left[\int_{x}^{\pi} q(t) d t\right] \cos [(n+2)(\pi-x)]+O\left(n^{-2} \eta(n)\right) .
\end{aligned}
$$

Now we give an example to illustrate our results. Let $q(t)=t^{-\frac{1}{2}}-2 \pi^{-\frac{1}{2}}$. Using (25)-(32), following estimates for $\Psi\left(x, \lambda_{n}\right)$ and $\Phi\left(x, \lambda_{n}\right)$ are obtained: $a_{4} \neq 0, \beta \neq 0$

$$
\begin{aligned}
& \Psi\left(x, \lambda_{n}\right)=a_{4}(n+1)^{2} \cos [(n+1) x]+(n+1) \\
& \times\left[a_{3}+a_{4}\left(x^{\frac{1}{2}}-\pi^{-\frac{1}{2}} x\right)\right] \sin [(n+1) x]+O(n \eta(n)), \\
& \Phi\left(x, \lambda_{n}\right)=\sin \beta \cos [(n+1)(\pi-x)]+\frac{1}{(n+1)} \\
& \times\left[\cos \beta+\sin \beta\left(-x^{\frac{1}{2}}+\pi^{-\frac{1}{2}} x\right)\right] \sin [(n+1)(\pi-x)]+O\left(n^{-1} \eta(n)\right) ;
\end{aligned}
$$




$$
\begin{aligned}
& a_{4} \neq 0, \beta=0 \\
& \begin{aligned}
\Psi\left(x, \lambda_{n}\right)= & a_{4} \frac{(2 n+3)^{2}}{4} \cos \left[\frac{(2 n+3) x}{2}\right]+\frac{(2 n+3)}{2} \\
& \quad \times\left[a_{3}+a_{4}\left(x^{\frac{1}{2}}-\pi^{-\frac{1}{2}} x\right)\right] \sin \left[\frac{(2 n+3) x}{2}\right]+O(n \eta(n)), \\
\Phi\left(x, \lambda_{n}\right)= & \frac{2}{(2 n+3)} \sin \left[\frac{(2 n+3)(\pi-x)}{2}\right]-\frac{2}{(2 n+3)^{2}} \\
& \quad \times\left(-2 x^{\frac{1}{2}}+2 \pi^{-\frac{1}{2}} x\right) \cos \left[\frac{(2 n+3)(\pi-x)}{2}\right]+O\left(n^{-2} \eta(n)\right) ;
\end{aligned}
\end{aligned}
$$

$$
\begin{aligned}
& a_{4}=0, \beta \neq 0 \\
& \begin{aligned}
\Psi\left(x, \lambda_{n}\right)=a_{3} \frac{(2 n+3)}{2} & \sin \left[\frac{(2 n+3) x}{2}\right] \\
- & {\left[a_{2}+a_{3}\left(x^{\frac{1}{2}}-\pi^{-\frac{1}{2}} x\right)\right] \cos \left[\frac{(2 n+3) x}{2}\right]+O(\eta(n)), }
\end{aligned}
\end{aligned}
$$$$
\Phi\left(x, \lambda_{n}\right)=\sin \beta \cos \left[\frac{(2 n+3)(\pi-x)}{2}\right]+\frac{2}{(2 n+3)}
$$$$
\times\left[\cos \beta+\sin \beta\left(-x^{\frac{1}{2}}+\pi^{-\frac{1}{2}} x\right)\right] \sin \left[\frac{(2 n+3)(\pi-x)}{2}\right]+O\left(n^{-1} \eta(n)\right) ;
$$$$
a_{4}=0, \beta=0
$$$$
\Psi\left(x, \lambda_{n}\right)=a_{3}(n+2) \sin [(n+2) x]
$$$$
-\left[a_{2}+a_{3}\left(x^{\frac{1}{2}}-\pi^{-\frac{1}{2}} x\right)\right] \cos [(n+2) x]+O(\eta(n)),
$$

$\Phi\left(x, \lambda_{n}\right)=\frac{1}{(n+2)} \sin [(n+2)(\pi-x)]-\frac{1}{(n+2)^{2}}\left(-x^{\frac{1}{2}}+\pi^{-\frac{1}{2}} x\right)$

$$
\times \cos [(n+2)(\pi-x)]+O\left(n^{-2} \eta(n)\right) .
$$

\section{The Method}

We associate with (1) the Riccati equation

$$
v^{\prime}=-\lambda+q-v^{2}
$$

We define

$$
S(t, \lambda):=\operatorname{Re}\{v(t, \lambda)\}
$$




$$
T(t, \lambda):=\operatorname{Im}\{v(t, \lambda)\} .
$$

It is shown in [3] that any real-valued solution of (1) is in the form

$$
y(t, \lambda)=R(t, \lambda) \cos (\theta(t, \lambda))
$$

with

$$
\frac{R^{\prime}(t, \lambda)}{R(t, \lambda)}=S(t, \lambda), \quad \theta^{\prime}(t, \lambda)=T(t, \lambda) .
$$

We consider (33) on $[a, b]$ and set

$$
v(t, \lambda):=i \lambda^{\frac{1}{2}}+\sum_{n=1}^{\infty} v_{n}(t, \lambda) .
$$

Substitution of (37) into (33) and rearrangement then gives

$$
\begin{aligned}
v_{1}^{\prime}+2 i \lambda^{\frac{1}{2}} v_{1}+v_{2}^{\prime}+2 i \lambda^{\frac{1}{2}} v_{2}+ & \sum_{n=3}^{\infty}\left(v_{n}^{\prime}+2 i \lambda^{\frac{1}{2}} v_{n}\right) \\
& =q-v_{1}^{2}-\sum_{n=3}^{\infty}\left(v_{n-1}^{2}+2 v_{n-1} \sum_{m=1}^{n-2} v_{m}\right) .
\end{aligned}
$$

We choose the $v_{n}$ so that

$$
\begin{aligned}
& v_{1}^{\prime}+2 i \lambda^{\frac{1}{2}} v_{1}=q, \\
& v_{2}^{\prime}+2 i \lambda^{\frac{1}{2}} v_{2}=-v_{1}^{2}, \\
& \ldots \ldots \ldots \ldots \ldots \ldots \ldots \ldots \ldots \ldots \ldots \ldots \ldots \ldots \ldots \ldots \ldots \ldots \ldots \\
& v_{n}^{\prime}+2 i \lambda^{\frac{1}{2}} v_{n}=-\left(v_{n-1}^{2}+2 v_{n-1} \sum_{m=1}^{n-2} v_{m}\right)
\end{aligned}
$$

for $n=3,4, \ldots$, and

$$
\begin{aligned}
& v_{1}(t, \lambda)=-e^{-2 i \lambda^{\frac{1}{2}} t} \int_{t}^{b} e^{2 i \lambda^{\frac{1}{2}} x} q(x) d x, \\
& v_{2}(t, \lambda)=e^{-2 i \lambda^{\frac{1}{2}} t} \int_{t}^{b} e^{2 i \lambda^{\frac{1}{2}} x} v_{1}^{2}(x, \lambda) d x, \ldots \ldots \ldots \ldots \ldots \ldots \ldots \ldots \ldots \ldots \ldots \ldots \ldots \ldots \ldots \ldots \ldots \ldots \ldots \ldots \ldots \ldots \ldots \ldots \ldots \ldots \\
& \ldots \ldots \ldots \ldots \ldots \\
& v_{n}(t, \lambda)=e^{-2 i \lambda^{\frac{1}{2}} t} \int_{t}^{b} e^{2 i \lambda^{\frac{1}{2}} x}\left(v_{n-1}^{2}+2 v_{n-1} \sum_{m=1}^{n-2} v_{m}\right) d x .
\end{aligned}
$$


It is proven in [3] that $\sum_{n=1}^{\infty} v_{n}^{\prime}(t, \lambda)$ is uniformly absolutely convergent and the series $i \lambda^{\frac{1}{2}}+\sum_{n=1}^{\infty} v_{n}(t, \lambda)$ is thus a solution of (33) and

$$
\begin{aligned}
& S(t, \lambda)=\operatorname{Re} \sum_{n=1}^{\infty} v_{n}(t, \lambda), \\
& T(t, \lambda)=\lambda^{\frac{1}{2}}+\operatorname{Im} \sum_{n=1}^{\infty} v_{n}(t, \lambda) .
\end{aligned}
$$

It is also proven in [3] that there exist a sequence $\left\{k_{n}\right\}$ of real numbers with

$$
\left|v_{n}(t, \lambda)\right| \leq k_{n} \eta(\lambda)^{n},
$$

(Lemma 2.2, [3]). It is shown in [7] that any nontrivial real-valued solution, $z$, of (1) can be expressed as

$$
z(x, \lambda)=c_{1} \exp \left(\int_{a}^{x} S(t, \lambda) d t\right) \cos \left\{c_{2}+\int_{a}^{x} T(t, \lambda) d t\right\}
$$

with

$$
\text { (44) } \begin{aligned}
z^{\prime}(x, \lambda)= & c_{1} S(x, \lambda) \exp \left(\int_{a}^{x} S(t, \lambda) d t\right) \cos \left\{c_{2}+\int_{a}^{x} T(t, \lambda) d t\right\} \\
& -c_{1} \exp \left(\int_{a}^{x} S(t, \lambda) d t\right) \sin \left(c_{2}+\int_{a}^{x} T(t, \lambda) d t\right) T(x, \lambda)
\end{aligned}
$$

where $S(x, \lambda)$ and $T(x, \lambda)$ are given by (34) and (35), respectively.

\section{Proof of the Results}

We first give the following lemma:

Lemma 4.1. As $\lambda \longrightarrow \infty$

(i)

$$
\frac{S(x, \lambda)}{T(x, \lambda)}=-\lambda^{-\frac{1}{2}} \sin \left(2 \lambda^{\frac{1}{2}} x+\zeta_{x}\right)+O\left(\lambda^{-\frac{1}{2}} \eta(\lambda)^{2}\right),
$$

(ii)

$$
\begin{aligned}
\frac{a_{3} \lambda-a_{1}}{\left(a_{4} \lambda-a_{2}\right) T(a, \lambda)} & =\frac{a_{3}}{a_{4}} \lambda^{-\frac{1}{2}}+O\left(\lambda^{-1} \eta(\lambda)\right), \\
\frac{a_{2} T(a, \lambda)}{a_{3} \lambda-a_{1}+a_{2} S(a, \lambda)} & =\frac{a_{2}}{a_{3}} \lambda^{-\frac{1}{2}}+O\left(\lambda^{-1} \eta(\lambda)\right),
\end{aligned}
$$


(iii)

(48)

$$
\begin{aligned}
& \int_{a}^{x} S(t, \lambda) d t \\
& \quad=\frac{1}{2 \lambda^{\frac{1}{2}}}\left\{\cos \left(2 \lambda^{\frac{1}{2}} x+\zeta_{x}\right)-\cos \left(2 \lambda^{\frac{1}{2}} a+\zeta_{a}\right)\right\}+O\left(\lambda^{-\frac{1}{2}} \eta(\lambda)^{2}\right)
\end{aligned}
$$

(iv)

$$
\begin{aligned}
& \int_{a}^{x} T(t, \lambda) d t=\lambda^{\frac{1}{2}}(x-a) \\
& -\frac{1}{2 \lambda^{\frac{1}{2}}}\left\{\sin \left(2 \lambda^{\frac{1}{2}} x+\zeta_{x}\right)-\sin \left(2 \lambda^{\frac{1}{2}} a+\zeta_{a}\right)+\int_{a}^{x} q(t) d t\right\}+O\left(\lambda^{-\frac{1}{2}} \eta(\lambda)^{2}\right),
\end{aligned}
$$

where

$$
\begin{aligned}
& \sin \zeta_{t}=\int_{t}^{b} q(t) \cos \left(2 \lambda^{\frac{1}{2}} t\right) d t, \\
& \cos \zeta_{t}=\int_{t}^{b} q(t) \sin \left(2 \lambda^{\frac{1}{2}} t\right) d t .
\end{aligned}
$$

\section{Proof.}

(i) It is shown in [2] that

$$
\begin{aligned}
& S(x, \lambda)=-\sin \left(2 \lambda^{\frac{1}{2}} x+\zeta_{x}\right)+O\left(\eta(\lambda)^{2}\right), \\
& T(x, \lambda)=\lambda^{\frac{1}{2}}-\cos \left(2 \lambda^{\frac{1}{2}} x+\zeta_{x}\right)+O\left(\eta(\lambda)^{2}\right) .
\end{aligned}
$$

Using this last two equalities we get

$$
\begin{aligned}
\frac{S(x, \lambda)}{T(x, \lambda)} & =\frac{-\sin \left(2 \lambda^{\frac{1}{2}} x+\zeta_{x}\right)+O\left(\eta(\lambda)^{2}\right)}{\lambda^{\frac{1}{2}}-\cos \left(2 \lambda^{\frac{1}{2}} x+\zeta_{x}\right)+O\left(\eta(\lambda)^{2}\right)} \\
& =-\frac{\sin \left(2 \lambda^{\frac{1}{2}} x+\zeta_{x}\right)+O\left(\eta(\lambda)^{2}\right)}{\lambda^{\frac{1}{2}}\left[1-\lambda^{-\frac{1}{2}} \cos \left(2 \lambda^{\frac{1}{2}} x+\zeta_{x}\right)+O\left(\lambda^{-\frac{1}{2}} \eta(\lambda)^{2}\right)\right]} \\
& =-\lambda^{-\frac{1}{2}} \sin \left(2 \lambda^{\frac{1}{2}} x+\zeta_{x}\right)+O\left(\lambda^{-\frac{1}{2}} \eta(\lambda)^{2}\right) .
\end{aligned}
$$

(ii) From (52) and (53) we obtain

$$
\begin{aligned}
\frac{a_{3} \lambda-a_{1}}{\left(a_{4} \lambda-a_{2}\right) T(a, \lambda)} & =\frac{a_{3} \lambda-a_{1}}{\left(a_{4} \lambda-a_{2}\right)\left[\lambda^{\frac{1}{2}}-\cos \left(2 \lambda^{\frac{1}{2}} a+\zeta_{a}\right)+O\left(\eta(\lambda)^{2}\right)\right]} \\
& =\frac{a_{3}}{a_{4}} \lambda^{-\frac{1}{2}}+O\left(\lambda^{-1} \eta(\lambda)\right)
\end{aligned}
$$


and

$$
\begin{aligned}
\frac{a_{2} T(a, \lambda)}{a_{3} \lambda-a_{1}+a_{2} S(a, \lambda)} & =\frac{a_{2}\left\{\lambda^{\frac{1}{2}}-\cos \left(2 \lambda^{\frac{1}{2}} a+\zeta_{a}\right)+O\left(\eta(\lambda)^{2}\right)\right\}}{a_{3} \lambda-a_{1}+a_{2}\left\{-\sin \left(2 \lambda^{\frac{1}{2}} a+\zeta_{a}\right)+O\left(\eta(\lambda)^{2}\right)\right\}} \\
& =\frac{a_{2}}{a_{3}} \lambda^{-\frac{1}{2}}+O\left(\lambda^{-1} \eta(\lambda)\right) .
\end{aligned}
$$

(iii) We now evaluate $\int_{a}^{x} S(t, \lambda) d t=\int_{a}^{x} \operatorname{Re}(v(t, \lambda)) d t$. From (39) and (42), we see that

$$
\begin{aligned}
\int_{a}^{x} S(t, \lambda) d t= & \int_{a}^{b} S(t, \lambda) d t-\int_{x}^{b} S(t, \lambda) d t \\
= & -\int_{a}^{b} \cos 2 \lambda^{\frac{1}{2}} t\left(\int_{t}^{b} \cos 2 \lambda^{\frac{1}{2}} x q(x) d x\right) d t \\
& -\int_{a}^{b} \sin 2 \lambda^{\frac{1}{2}} t\left(\int_{t}^{b} \sin 2 \lambda^{\frac{1}{2}} x q(x) d x\right) d t \\
& +\int_{x}^{b} \cos 2 \lambda^{\frac{1}{2}} t\left(\int_{t}^{b} \cos 2 \lambda^{\frac{1}{2}} x q(x) d x\right) d t \\
& +\int_{x}^{b} \sin 2 \lambda^{\frac{1}{2}} t\left(\int_{t}^{b} \sin 2 \lambda^{\frac{1}{2}} x q(x) d x\right) d t \\
& +O\left(\lambda^{-\frac{1}{2}} \eta(\lambda)^{2}\right) .
\end{aligned}
$$

Using a change of the order of integration and integration by parts

$$
\begin{aligned}
\int_{a}^{x} S(t, \lambda) d t=- & \int_{a}^{b} \cos 2 \lambda^{\frac{1}{2}} x q(x)\left[\frac{\sin 2 \lambda^{\frac{1}{2}}(x-a)}{2 \lambda^{\frac{1}{2}}}\right] d x \\
& -\int_{a}^{b} \sin 2 \lambda^{\frac{1}{2}} x q(x)\left[-\frac{\cos 2 \lambda^{\frac{1}{2}}(x-a)}{2 \lambda^{\frac{1}{2}}}\right] d x \\
& -\frac{\sin 2 \lambda^{\frac{1}{2}} x}{2 \lambda^{\frac{1}{2}}} \int_{x}^{b} \cos 2 \lambda^{\frac{1}{2}} x q(x) d x \\
& +\frac{\cos 2 \lambda^{\frac{1}{2}} x}{2 \lambda^{\frac{1}{2}}} \int_{x}^{b} \sin 2 \lambda^{\frac{1}{2}} x q(x) d x+O\left(\lambda^{-\frac{1}{2}} \eta(\lambda)^{2}\right) \\
= & \frac{1}{2 \lambda^{\frac{1}{2}}}\left\{\cos \left(2 \lambda^{\frac{1}{2}} x+\zeta_{x}\right)-\cos \left(2 \lambda^{\frac{1}{2}} a+\zeta_{a}\right)\right\} \\
& +O\left(\lambda^{-\frac{1}{2}} \eta(\lambda)^{2}\right) .
\end{aligned}
$$


The last equality follows from some algebraic calculations.

(iv) Similarly we evaluate $\int_{a}^{x} T(t, \lambda) d t=\int_{a}^{x} \operatorname{Im}(v(t, \lambda)) d t$. From (39) and (42), we see that

$$
\begin{aligned}
\int_{a}^{x} T(t, \lambda) d t= & \int_{a}^{b} T(t, \lambda) d t-\int_{x}^{b} T(t, \lambda) d t \\
= & \int_{a}^{x} \lambda^{\frac{1}{2}} d t+\int_{a}^{b} \sin 2 \lambda^{\frac{1}{2}} t\left(\int_{t}^{b} \cos 2 \lambda^{\frac{1}{2}} x q(x) d x\right) d t \\
& \quad-\int_{a}^{b} \cos 2 \lambda^{\frac{1}{2}} t\left(\int_{t}^{b} \sin 2 \lambda^{\frac{1}{2}} x q(x) d x\right) d t \\
& -\int_{x}^{b} \sin 2 \lambda^{\frac{1}{2}} t\left(\int_{t}^{b} \cos 2 \lambda^{\frac{1}{2}} x q(x) d x\right) d t \\
& +\int_{x}^{b} \cos 2 \lambda^{\frac{1}{2}} t\left(\int_{t}^{b} \sin 2 \lambda^{\frac{1}{2}} x q(x) d x\right) d t \\
& +O\left(\lambda^{-\frac{1}{2}} \eta(\lambda)^{2}\right) \\
= & \lambda^{\frac{1}{2}}(x-a)-\frac{1}{2 \lambda^{\frac{1}{2}}}\left\{\sin \left(2 \lambda^{\frac{1}{2}} x+\zeta_{x}\right)-\sin \left(2 \lambda^{\frac{1}{2}} a+\zeta_{a}\right)\right. \\
& \left.+\int_{a}^{x} q(t) d t\right\}+O\left(\lambda^{-\frac{1}{2}} \eta(\lambda)^{2}\right) .
\end{aligned}
$$

The last equality follows from a change of the order of integration and integration by parts.

Proof of Theorem 2.1 .

(i) For $a_{4} \neq 0$; from (4), (43) and (44) we obtain

$$
\begin{aligned}
\Psi(a, \lambda) & =c_{1} \cos c_{2}=a_{4} \lambda-a_{2}, \\
\Psi^{\prime}(a, \lambda) & =c_{1} S(a, \lambda) \cos c_{2}-c_{1} \sin c_{2} T(a, \lambda)=a_{3} \lambda-a_{1} .
\end{aligned}
$$

From the last two equalities

$$
\begin{aligned}
& c_{1}=\frac{a_{4} \lambda-a_{2}}{\cos c_{2}}, \\
& c_{2}=\tan ^{-1}\left[F_{1}(a, \lambda)\right] .
\end{aligned}
$$

Hence

$$
c_{1}=\frac{a_{4} \lambda-a_{2}}{\cos \left\{\tan ^{-1}\left[F_{1}(a, \lambda)\right]\right\}} .
$$


Substitution the values of $c_{1}$ and $c_{2}$ in (43) proves part (i).

(ii) For $a_{4}=0$; again using (54) and (55) we obtain

$$
\begin{aligned}
& c_{1}=-\frac{a_{2}}{\cos c_{2}}, \\
& c_{2}=\cot ^{-1}\left[F_{2}(a, \lambda)\right] .
\end{aligned}
$$

So

$$
c_{1}=-\frac{a_{2}}{\cos \left\{\cot ^{-1}\left[F_{2}(a, \lambda)\right]\right\}} .
$$

Substitution the values of $c_{1}$ and $c_{2}$ in (43) proves part (ii).

Proof of Theorem 2.2.

(i) For $\beta \neq 0$; from (5), (43) and (44) we obtain

$$
\Phi(b, \lambda)=c_{1} \exp \left(\int_{a}^{b} S(t, \lambda) d t\right) \cos \left\{c_{2}+\int_{a}^{b} T(t, \lambda) d t\right\}=\sin \beta,
$$

(60) $\Phi^{\prime}(b, \lambda)=c_{1} S(b, \lambda) \exp \left(\int_{a}^{b} S(t, \lambda) d t\right) \cos \left\{c_{2}+\int_{a}^{b} T(t, \lambda) d t\right\}$

$$
\begin{aligned}
& -c_{1} \exp \left(\int_{a}^{b} S(t, \lambda) d t\right) \sin \left\{c_{2}+\int_{a}^{b} T(t, \lambda) d t\right\} T(b, \lambda) \\
= & -\cos \beta .
\end{aligned}
$$

From (59) and (60)

$$
\begin{aligned}
& c_{1}=\frac{\sin \beta}{\exp \left(\int_{a}^{b} S(t, \lambda) d t\right) \cos \left\{c_{2}+\int_{a}^{b} T(t, \lambda) d t\right\}}, \\
& c_{2}=\tan ^{-1}\left\{\frac{S(b, \lambda)+\cot \beta}{T(b, \lambda)}\right\}-\int_{a}^{b} T(t, \lambda) d t .
\end{aligned}
$$

Hence

$$
c_{1}=\frac{\sin \beta}{\exp \left(\int_{a}^{b} S(t, \lambda) d t\right) \cos \left\{\tan ^{-1}\left[\frac{S(b, \lambda)+\cot \beta}{T(b, \lambda)}\right]\right\}} .
$$

Substitution of $c_{1}$ and $c_{2}$ in (43) proves part (i).

(ii) For $\beta=0$, again from (5), (43) and (44) we obtain

(64) $\Phi(b, \lambda)=c_{1} \exp \left(\int_{a}^{b} S(t, \lambda) d t\right) \cos \left\{c_{2}+\int_{a}^{b} T(t, \lambda) d t\right\}=0$, 
(65)

$$
\begin{aligned}
\Phi^{\prime}(b, \lambda)= & c_{1} S(b, \lambda) \exp \left(\int_{a}^{b} S(t, \lambda) d t\right) \cos \left\{c_{2}+\int_{a}^{b} T(t, \lambda) d t\right\} \\
& -c_{1} \exp \left(\int_{a}^{b} S(t, \lambda) d t\right) \sin \left\{c_{2}+\int_{a}^{b} T(t, \lambda) d t\right\} T(b, \lambda) \\
= & -1
\end{aligned}
$$

Using (64) we see that

$$
c_{2}=\frac{\pi}{2}-\int_{a}^{b} T(t, \lambda) d t .
$$

Hence from (65) and (66)

$$
c_{1}=\frac{1}{\exp \left(\int_{a}^{b} S(t, \lambda) d t\right) T(b, \lambda)} .
$$

Substitution of $c_{1}$ and $c_{2}$ in (43) proves part (ii).

Proof of Theorem 2.3.

(i) For $a_{4} \neq 0$, we evaluate the terms in (6). Firstly, substituting (45) and (46) into $F_{1}(a, \lambda)$ given by (7) we obtain

$$
F_{1}(a, \lambda)=-\lambda^{-\frac{1}{2}}\left[\sin \left(2 \lambda^{\frac{1}{2}} a+\zeta_{a}\right)+\frac{a_{3}}{a_{4}}\right]+O\left(\lambda^{-\frac{1}{2}} \eta(\lambda)^{2}\right),
$$

hence

$$
\tan ^{-1}\left[F_{1}(a, \lambda)\right]=-\frac{a_{3}}{a_{4}} \lambda^{-\frac{1}{2}}+O\left(\lambda^{-\frac{1}{2}} \eta(\lambda)\right) .
$$

From (57) we know that

$$
c_{2}=\tan ^{-1}\left[F_{1}(a, \lambda)\right]=-\frac{a_{3}}{a_{4}} \lambda^{-\frac{1}{2}}+O\left(\lambda^{-\frac{1}{2}} \eta(\lambda)\right)
$$

and from that

$$
\begin{aligned}
\cos c_{2} & =1-\frac{1}{2}\left(\frac{a_{3}}{a_{4}}\right)^{2} \lambda^{-1}+O\left(\lambda^{-1} \eta(\lambda)\right), \\
\sin c_{2} & =-\frac{a_{3}}{a_{4}} \lambda^{-\frac{1}{2}}+O\left(\lambda^{-\frac{1}{2}} \eta(\lambda)\right), \\
\frac{a_{4} \lambda-a_{2}}{\cos c_{2}} & =a_{4} \lambda+O(1) .
\end{aligned}
$$


Using $\int_{a}^{x} S(t, \lambda) d t$ given by (48) we get

(74)

$$
\begin{aligned}
& \exp \left(\int_{a}^{x} S(t, \lambda) d t\right) \\
& \quad=1+\frac{1}{2 \lambda^{\frac{1}{2}}}\left\{\cos \left(2 \lambda^{\frac{1}{2}} x+\zeta_{x}\right)-\cos \left(2 \lambda^{\frac{1}{2}} a+\zeta_{a}\right)\right\}+O\left(\lambda^{-\frac{1}{2}} \eta(\lambda)^{2}\right)
\end{aligned}
$$

and also, using $\int_{a}^{x} T(t, \lambda) d t$ given by (49)

$$
\begin{aligned}
& \sin \left(\int_{a}^{x} T(t, \lambda) d t\right) \\
& =\sin \left[\lambda^{\frac{1}{2}}(x-a)-\frac{1}{2 \lambda^{\frac{1}{2}}} \int_{a}^{x} q(t) d t\right]+O\left(\lambda^{-\frac{1}{2}} \eta(\lambda)\right)
\end{aligned}
$$

(76) $\cos \left(\int_{a}^{x} T(t, \lambda) d t\right)$

$$
=\cos \left[\lambda^{\frac{1}{2}}(x-a)-\frac{1}{2 \lambda^{\frac{1}{2}}} \int_{a}^{x} q(t) d t\right]+O\left(\lambda^{-\frac{1}{2}} \eta(\lambda)\right) .
$$

Hence

$$
\begin{aligned}
\cos \left[c_{2}+\right. & \left.\int_{a}^{x} T(t, \lambda) d t\right]=\cos \left[\lambda^{\frac{1}{2}}(x-a)-\frac{1}{2 \lambda^{\frac{1}{2}}} \int_{a}^{x} q(t) d t\right] \\
& +\frac{a_{3}}{a_{4}} \lambda^{-\frac{1}{2}} \sin \left[\lambda^{\frac{1}{2}}(x-a)-\frac{1}{2 \lambda^{\frac{1}{2}}} \int_{a}^{x} q(t) d t\right]+O\left(\lambda^{-\frac{1}{2}} \eta(\lambda)\right) .
\end{aligned}
$$

Substituting the values of (73), (74) and (77) into (6) and using trigonometric expansions we prove part (i). The other part (ii) can be proved similarly.

Proof of Theorem 2.4.

(i) For $\beta \neq 0$, we evaluate the terms in (10). Using (45) and (53), $F_{3}(b, \lambda)$ given by (11) is obtained as

$$
\begin{aligned}
F_{3}(b, \lambda) & =\frac{S(b, \lambda)+\cot \beta}{T(b, \lambda)}=\frac{\cot \beta+O\left(\eta(\lambda)^{2}\right)}{\lambda^{\frac{1}{2}}+O\left(\eta(\lambda)^{2}\right)} \\
& =\frac{\cot \beta+O\left(\eta(\lambda)^{2}\right)}{\lambda^{\frac{1}{2}}\left[1+O\left(\lambda^{-\frac{1}{2}} \eta(\lambda)^{2}\right)\right]}=\lambda^{-\frac{1}{2}} \cot \beta+O\left(\lambda^{-\frac{1}{2}} \eta(\lambda)^{2}\right)
\end{aligned}
$$

and from that

$$
\begin{aligned}
\tan ^{-1}\left[F_{3}(b, \lambda)\right] & =\lambda^{-\frac{1}{2}} \cot \beta+O\left(\lambda^{-\frac{1}{2}} \eta(\lambda)^{2}\right), \\
\cos \left\{\tan ^{-1}\left[F_{3}(b, \lambda)\right]\right\} & =1-\frac{1}{2} \lambda^{-1} \cot ^{2} \beta+O\left(\lambda^{-1} \eta(\lambda)^{2}\right) .
\end{aligned}
$$


Using $\int_{a}^{x} S(t, \lambda) d t$ given by (48) we get

$$
\exp \left(\int_{a}^{b} S(t, \lambda) d t\right)=1+O\left(\lambda^{-\frac{1}{2}} \eta(\lambda)\right)
$$

Hence

$$
\begin{aligned}
& \frac{\sin \beta}{\exp \left(\int_{a}^{b} S(t, \lambda) d t\right) \cos \left\{\tan ^{-1}\left[F_{3}(b, \lambda)\right]\right\}} \\
& =\frac{\sin \beta}{1+O\left(\lambda^{-\frac{1}{2}} \eta(\lambda)\right)}=\sin \beta+O\left(\lambda^{-\frac{1}{2}} \eta(\lambda)\right) .
\end{aligned}
$$

Also, using $\int_{a}^{x} T(t, \lambda) d t$ given by (49)

$$
\begin{aligned}
\int_{x}^{b} T(t, \lambda) d t & =\int_{a}^{b} T(t, \lambda) d t-\int_{a}^{x} T(t, \lambda) d t=\lambda^{\frac{1}{2}}(b-x) \\
+ & \frac{1}{2 \lambda^{\frac{1}{2}}}\left\{\sin \left(2 \lambda^{\frac{1}{2}} x+\zeta_{x}\right)-\int_{x}^{b} q(t) d t\right\}+O\left(\lambda^{-\frac{1}{2}} \eta(\lambda)^{2}\right) .
\end{aligned}
$$

From the last equality and $\tan ^{-1}\left[F_{3}(b, \lambda)\right]$ given by (79) we see that

$$
\begin{aligned}
\cos \left\{\tan ^{-1}\left[F_{3}(b, \lambda)\right]-\int_{x}^{b} T(t, \lambda) d t\right\} \\
=\cos \left[\lambda^{\frac{1}{2}}(b-x)-\frac{1}{2 \lambda^{\frac{1}{2}}} \int_{x}^{b} q(t) d t\right] \\
\quad+\lambda^{-\frac{1}{2}} \cot \beta \sin \left[\lambda^{\frac{1}{2}}(b-x)-\frac{1}{2 \lambda^{\frac{1}{2}}} \int_{x}^{b} q(t) d t\right]+O\left(\lambda^{-\frac{1}{2}} \eta(\lambda)\right) .
\end{aligned}
$$

Finally, substituting the values of (74), (82) and (84) into (10) and using trigonometric expansions we prove part (i).

(ii) For $\beta=0$, we evaluate the terms in (12). From (53) and (81) we obtain

$$
\frac{1}{\exp \left(\int_{a}^{b} S(t, \lambda) d t\right) T(b, \lambda)}=\frac{1}{\lambda^{\frac{1}{2}}+O(\eta(\lambda))}=\lambda^{-\frac{1}{2}}+O\left(\lambda^{-1} \eta(\lambda)\right) .
$$

Also, using $\int_{x}^{b} T(t, \lambda) d t$ given by (83) we see that

$$
\begin{aligned}
\cos \left[\frac{\pi}{2}-\int_{x}^{b} T(t, \lambda) d t\right] & =\sin \left[\int_{x}^{b} T(t, \lambda) d t\right] \\
& =\sin \left[\lambda^{\frac{1}{2}}(b-x)-\frac{1}{2 \lambda^{\frac{1}{2}}} \int_{x}^{b} q(t) d t\right]+O\left(\lambda^{-\frac{1}{2}} \eta(\lambda)\right) .
\end{aligned}
$$


Finally, substituting the values of (74), (85) and (86) into (12) and using trigonometric expansions we prove part (ii).

To prove Theorem 2.5 and Theorem 2.6 we use eigenvalues obtained by [2] previously together with Theorem 2.3 and Theorem 2.4 .

\section{REFERENCES}

1. Bauer, W. F., Modified Sturm-Liouville systems, Quart. Appl. Math. 11 (1953), 273-283.

2. Coşkun, H., and Başkaya, E., Asymptotics of eigenvalues of regular Sturm-Liouville problems with eigenvalue parameter in the boundary condition for integrable potential, Math. Scand. 107 (2010), 209-223.

3. Coskun, H., and Harris, B. J., Estimates for the periodic and semi-periodic eigenvalues of Hill's equation, Proc. Roy. Soc. Edin. Sect. A 130 (2000), 991-998.

4. Coşkun, H., Asymptotic approximations of eigenvalues and eigenfunctions for regular SturmLiouville problems, Rocky Mountain J. Math, 36 (2006), 867-883.

5. Fulton, C. T., Two point boundary value problems with eigenvalue parameter contained in the boundary conditions, Proc. Roy. Soc. Edin. Sect. A 77 (1977), 293-308.

6. Gaskell, R. E., A problem in heat conduction and an expansion theorem, Amer. J. Math. 64 (1942), 447-455.

7. Harris, B. J., The form of the spectral functions associated with Sturm-Liouville problems with continuous spectrum, Mathematika 44 (1997), 149-161.

8. Langer, R. E., A problem in diffusion or in the flow of heat for a solid in contact with a fluid, Tohoku Math. J. 35 (1932), 360-375.

9. Morgan, G. W., Some remarks on a class of eigenvalue problems with special boundary conditions, Quart. Appl. Math. 11 (1953), 157-165.

10. Peddie, W., Note on the cooling of a sphere in a mass of well stirred liquid, Proc. Edinburgh Math. Soc. 19 (1901), 34-35.

11. Peek, R. L., Jr., Solutions to a problem in diffusion employing a non-orthogonal sine series, Ann. of Math. 30 (1929), 265-269.

12. Poisson, S. D., Memoire sur la Maniere d'exprimer les Fonctions par des Series de quantites periodiques, et sur l'Usage de cette Transformation dans la Resolution de differens Problemes, J. Ecole Polytechnique 18 (1820), 417-489.

13. Schneider, A., Zur Einordnung selbstadjungierter Rand-eigenwertprobleme bei gewöhnlichen differentialgleichungen in die theorie S-hermitescher Rand-eigenwertprobleme, Math. Ann. 178 (1968), 277-294.

14. Titchmarsh, E. C., Eigenfunction expansions associated with second order differential equations I (2nd. ed.), Oxford Univ. Press, Oxford 1962.

15. Wagner, K. W., Elektromagnetische Ausgleichsvorgange in Freileitungen und Kabeln, Teubner, Leibzig 1908.

16. Walter, J., Regular eigenvalue problems with eigenvalue parameter in the boundary conditions, Math. Z. 133 (1973), 301-312.

DEPARTMENT OF MATHEMATICS

KARADENIZ TECHNICAL UNIVERSITY

TR-61080 TRABZON

TURKEY

E-mail: haskiz@ktu.edu.tr akabatas@ktu.edu.tr 